\section{RADIUM-ITS EXTRAORDINARY PROPERTIES}

In 1896 Prof. Henri Becquerel, of the Conservatoire des Arts et Métiers at Paris, discovered the radio-activity of uranium. He found that all com pounds of uranium, as well as the metal itself, continually emit radiations, which act upon photographi plates and have a penetrating power similar to that
of the X-rays. This was one of the first of a series of quite remarkable discoveries. Investigators im mediately experimented with various materials witl the hope that they might find other substances having the same property as uranium. Of the elements already known, thorium as well as uranium was found to be radio-active. But research has led to the discovery of three new radio-active substances, which are looked upon as new elements. These are radium, polonium, and actinium. Of these radium alone has been obtained in a pure condition, and it is the one which has been most experimented with

Prof. Curie, of the Ecole de Physique et de Chimie Industrielles at Paris, and Madame Curie turned their attention to pitchblende, a mineral which consists largely of oxides of uranium. They found that some samples of this mineral from Bohemia possessed a greater activity than either uranium or thorium, the greater activity than either uranium or thcrium, the
only substances then known to be radio-active. This fact led them to the conclusion that the activity of the pitchblende must be due to some new element of great activity. In order to find this new substance, they dissolved a quantity of pitchblende in acids and by the ordinary chemical methods, separated the material into portions containing different elements. They then observed which of these portions possessed
radio-activity. This could be done by exposing photographic plates wrapped in opaque paper to the substances and observing which plates were acted upon. But it could be done more expediently by anothe method. Becquerel had observed that the new radia tions-"Becquerel rays" as they are now calledrender the air through which they pass a conductor effect upon many other substances which do not ordinarily conduct electricity. The Curies had but to narily conduct electricity. The Curies had but to
measure the conducting power of the air in the im measure the conducting power of the air in the im-
mediate neighborhood of the material under investigation, to find whether the material was radio-active and to obtain a measure of its activity, if it possesse any. Guided by such experiments, they gradually concentrated the active substances into small portions of the material. One portion they believed to contain a new element, which they called "polonium;" an other yielded radium.

The radium greatly resembled barium chemically, and its separation from barium was the last and most difficult part of the operation. It was at length accomplished by fractional crystallizations and fractional precipitations, and in 1902 Madame Curie announced the preparation of pure radium chloride. E. Demar çay examined the spectrum of this material, and found that it consisted of lines which were not those of any previously known element, thus proving quite conpreviously known element, thus proving quite con-
clusively that the radium was actually a new element. Something about the rarity and the cost of radium may not be without interest. According to Prof. Curie, there are not two pounds of radium in existence. In the last three years not more than one and one quarter pounds have been manufactured. Even this small quantity is of all grades of purity. Absolutely pure radium dues not exist as a metal. Only its salts ar known. The substance with which chemists experi-
ment is chloride of radium associate with barium ment is chloride of radium associated with barium.
Of the value of radium many fantastic accounts have been given. It goes without saying that so rare a substance is costly-more precious indeed than an precious stone. Prof. Curie has in his possession what is probably the only pure specimen of chemi cally pure radium in the world. The sample is about the size of a buckshot, weighs not quite half a grain So many tons of pitchblende were required for the re production of this small amount that Prof. Curie has said it could not be bought for $\$ 20,000$. Indee such a specimen of radium has almost any commer cial value its possessor chooses to give to it. A firm of manufacturing chemists of Paris furnish tiny tubes of radium of a lower grade, containing an appreciable quantity of barium, and weighing about as much as Prof. Curie's precious specimen, for $\$ 5,000$. Preparations containing barium salts and small quantitie of radium are on the market at the much lower price of radium are on the market at the much lower price
of $\$ 4.50$ to $\$ 100$ per gramme, the gramme being equal to 15.42 grains.

The amount of radium contained in pitchbrende is so small that it must be brought to a concentration no less than five thousand times as great before it can be detected by that exceedingly delicate instrument the spectroscope. It is needless to say that the discovery of some mineral yielding radium in greater
quantities, is much to be desired. Sir William quantities, is much to be desired. Sir William
Crockes, reasoning from the facts that radium is very similar chemically to barium, and that elements of similar nature are likely to be associated in minerals,
experimented with a number of specimens of barium minerals with the hope of finding radium in them; but them were radio-active.

Radium has never been prepared in the metallic state. The radio-activity of the pure salts is very
great. Prof Curie states that it is a million times great. Prof. Curie states that it is a million times
as great as that of uranium. The radium rays will act upon a photographic plate in a few seconds, while uranium requires hours.

The radiations themselves are very interesting. They cannot be refracted, polarized, or regularly re lected, as ordinary light can be. They are quite different from light. Prof. Becquerel observed that a
part of them are deflected by a magnet. This impart of them are deflected by a magnet. This im
mediately reminds one of the cathode rays of Crcokes tube, which are similarly deflected. The cathode rays are now known to be nothing less than streams of most minute particles, carrying negative electricity and moving with enormous velocities. All evidence points to the deflectable portion of the Becquerel rays being quite the same thing. The Curies have shown that they also carry negative electricity; and Prof. Becquerel has shown that, like the cathode rays, they are deflected by electrostatic forces. From calculate the velocity of these particles. They do not all move at quite the same rate. A portion of not all move at quite the same rate. A portion of them have a velocity of 100,000 miles per second, a
velocity quite comparable with that of light, 186,000 miles per second. The cathode rays in a Crookes tub have a velocity of about two-thirds that of light.

Prof. Becquerel has also calculated the ratio of the mass of the particles to the quantity of electricity which they carry, and this too has about the same Thomson has shown that the particles in a Crookes tube have a mass only about one-thousandth of that of a hydrogen atcm, which we have always looked upon as the smallest particle of matter existing. We have reason to believe that the particles of the Becquere rays are of the same size

One might reasonably inquire whether radium does not rapidly lose weight as the result of the constant emission of these particles; but Prof. Becquerel has calculated that one square centimeter of radium sur-
face would lose only 1.2 milligrammes of matter in a thousand million years. However, A. Heyd weiller has recently found that radium does lose weight percept ibly. He found that 5 grammes of a material containing a small percentage of radium lost about 0.02
milligramme per day, and he observed a total loss of about $1 / 2$ milligramme.

The portion of the Becquerel rays which are not de flected by a magnet, appear to consist largely of very penetrating rays resembling the X-rays; but there are also rays of a third kind, easily absorbed.

One of the nost striking properties of radium is its luminosity. The pure radium chloride emit enough light to enable one to distinguish printed char acters. The rays from radium excite phosphorescence even common salt. The luminosity of radium is perhaps but the phosphorescence produced by its own haps but the phosphorescence produced by its own
rays. If a small quantity of radium is held against rays. If a small quantity of radium is held against
the forehead while the eyes are closed, one will see the forehead while the eyes are closed, one will see
light. The rays penetrate to the retina, and cause it to phosphoresce.

Certain chemical changes are brought about by the rays from radium. Under their influence, oxygen is converted into ozone, yellow phosphorus into red

The physiological action of the rays is quite mark ed. If a small quantity of radium be kept near the skin for a few hours, the rays produce a serious sore Prof. Becquerel once slipped a small quantity of radium contained in a glass tube into his vest pocket. He carried it in all about six hours. For some days no result was observed, but at length a sore developed which required seven weeks to heal. The hands of persons working with radium are likely to be affected. The fingers become inflamed and very painful. Prof Curie has said that he would not venture into a room
containing one kilogramme of radium, as it would containing one kilogramme of radium, as it would
probably destroy his eyesight, burn off his skin, and probably destroy
cven kill him.

E. Aschkinass and W. Caspari have exposed culture of a species of bacteria, Micrococcus prodigiosus, to the rays from radium, with the result that the bacteria were killed. It was necessary to place the radium que to those of the rays which are easily absorbed by due to tho
the air.

When any body is placed near to a radium salt ex posed to the air, it becomes radio-active itself. Thi induce activity is only temporary, however. It disappears in the course of a few hours or days. It does not depend upon the nature of the body in which it is induced. Even the hands and clothing of the experimenter become temporarily active. The induce activity seems to be produced not by the radiations, but by a radio-active "emanation" or gas-like substance
which is given off by radium and carried by the air. Exactly what this emanation is, is not known; but Prof. Rutherford and Miss Brookes have made a deter- mination of its rate of diffusion, which indicates that its molecular weight lies between 40 and 100 . F. Giesel states that a solution of radium bromide decomposes to some extent, with the liberation of bromine and the formation of radium hydroxide and other compounds; and that it also liberates a peculiar colorless gas which is radio-active. What this gas is has not
yet been made known. It may be mentioned that yet been made known. It may be mentioned that which is given off by thorium compounds has the chemical inertness of the gases of the argon group.

The energy of the rays from radium has been found to be quite considerable. Rutherford and MicClung have estimate that a gramme of radium radiates in a year energy equivalent to 3,000 gramme-calories, which is about one foot-pound per hour, that is, the power necessary to raise a pound a foot in an hour. The source of this energy is a mystery. Several theories have been presented to account for it. Rutherford and McClung suggest that the energy is liberated by the breaking down of the atoms into smaller particles, the particles that are radiated.

Radium is not likely to find much practical use soon, although it has been made use of in a new electroscope for investigating the electrical condition of the atmosphere; but the properties of radium are very important from a theoretical standpoint, for they promise to give us much information concerning the deeper nature of matter. Columbia University.

PROPOSED SUBWAY AND ELEVATED EXTENSION IN NEW YORK CITY

In a report recently submitte to the Rapid Transit Commissioners, the Chief Engineer of the Commission William Barclay Parsons, recommends that the Commission authorize the construction of a scheme of new subways, and extensions of the elevated railroad system, which will add enormously to the rapid tran-
sit facilities in this city. We give herewith a brief digest of the proposed work, and in a subsequent issue, we hope to publish plans and a more detailed description. The new subways include a two-track line from 42d Street and Broadway, south beneath Broadway to 14th Street, and thence below University Place, Worcester and Church Streets to South Ferry. A
short spur will run from Broadway under 32d Street to the new Pennsylvania terminus at Seventh Avenue Another line with three tracks would extend from the subway at 40th Street and Park Avenue, below Lexbe to carry a line from West Farms along the east side of Bronx Park to Wakefield and Mount Vernon.

During the construction of the subways, it is proposed to build the following additional elevated lines and tracks in the city: Two additional tracks on Second Avenue from the Harlem River to Chathan Square and over the Park Row line to City Hall; a third track from 59th Street to Ninth Avenue on the Third Avenue line, and a third track from south of sion of the Sixth Avenue line along Christophe Street to Greenwich Street on the Ninth Avenue line and the laying of a third track on the Sixth Avenue structure from Eighth Street northward; the layin of a third track from 14th Street on the Ninth Avenue line south to Cortland Street; the extension of the third track on the Ninth Avenue line from 116th Street north to 155th Street; the widening of the Putnam Railroad bridge across the Harlem to a three nue line with three tracks by way of Jerome Avenue to Woodlawn, and thence to a connection with the to Woodlawn, and thence to a connection with the
Putnam Railroad in Van Cortland Park; the abolishing of the surface tracks of the New York Central Railroad below 59th Street on the west side of Man hattan, and the substitution therefor of a four-track elevated freight and passenger viaduct, which will extend ky vay of West Street to Battery Place. The liminary investigations, is from $\$ 45,000,000$ to $\$ 50$, liminary

\section{THE CURRENT SUPPLEMENT.}

The current Supplemext, No. 1417, offers a wide variety of instructive articles. Mr. Frank C. Perkins describes some German and English tank locomotives, illustrating his text with photographs of engines. The English correspondent of the Scientific American continues his discussion of London's water supply. Electrical subjects are treated in articles on "The Theory of Wehnelt Interrupters," and the "Braun Portable Wireless Telegraph Equipments in the German Army." Mr. Henry R. Lordly's valuable paper on "Anti-Friction Bearings" is conciuded. Of technological interest is an article on "Improvement in Sugar Refining During the Last Twenty-five Years." The employment of balata as a substitute for gutta-percha Mr. Fre T Tane continues his "Naval War Game" series, the present installment being a continuation series, the present installment being a continuation
of the account of the imaginary battle between an American and a German fleet. 